\title{
Novel EGFR-bispecific recombinant immunotoxin based on cucurmosin shows potent anti-tumor efficiency in vitro
}

\author{
CAIYUN ZHANG ${ }^{1 *}$, YUMEI CAI $^{1,2^{*}}$, XIAOXUE DAI $^{1 *}$, JINGYU WU $^{1}$, YINXIANG LAN $^{1}$, \\ HUAJIN ZHANG ${ }^{1}$, MENGNI LU ${ }^{1}$, JUNHONG LIU ${ }^{1}$ and JIEMING XIE ${ }^{1}$ \\ ${ }^{1}$ Department of Pharmacology, School of Pharmacy, Fujian Provincial Key Laboratory of Natural Medicine Pharmacology, \\ Fujian Medical University, Fuzhou, Fujian 350122; ${ }^{2}$ Department of Pharmacy, \\ Haicang Hospital, Xiamen, Fujian 361026, P.R. China
}

Received April 17, 2020; Accepted October 9, 2020

DOI: $10.3892 /$ or.2020.7878

\begin{abstract}
Epidermal growth factor receptor (EGFR) is overexpressed in various tumors and is associated with cancer initiation, progression, and poor prognosis. Despite the achievements made by tyrosine kinase inhibitors and monoclonal antibodies in certain cases, many patients have not benefited from such treatment due to resistance. Immunotoxins (ITs) are antibody-cytotoxin chimeric molecules with specific cell killing ability, which have achieved different degrees of success in the treatment of a wide range of cancers in clinical trials. The aim of the current study was to examine a novel targeting EGFR recombinant immunotoxin Bs/cucurmosin (CUS) generated by fusing CUS to the EGFR-specific nanobody 7D12-9G8. Bs/CUS was successfully expressed in Escherichia coli strain BL21 (DE3) in a soluble form. Furthermore, it retained binding capacity and specificity with EGFR and was superior to rE/CUS, a monospecific IT we reported previously. In vitro results showed that Bs/CUS could be internalized into the cytoplasm and selectively kill cells in the picomolar range. Flow cytometry showed that Bs/CUS killed the cells mediated by the apoptosis pathway. Taken together, results of the current study indicated that Bs/CUS is a promising candidate that should be further evaluated as a cancer therapeutic for the treatment of EGFR-positive tumors.
\end{abstract}

\section{Introduction}

The epidermal growth factor receptor (EGFR) family is a transmembrane protein receptor with tyrosine kinase

Correspondence to: Professor Jieming Xie, Department of Pharmacology, School of Pharmacy, Fujian Provincial Key Laboratory of Natural Medicine Pharmacology, Fujian Medical University, 1 Xueyuan Road, Fuzhou, Fujian 350122, P.R. China

E-mail: jmxie@fjmu.edu.cn

*Contributed equally

Abbreviation: EGFR, epidermal growth factor receptor

Key words: cucurmosin, EGFR, nanobody, recombinant immunotoxin, targeted therapy activity $(1,2)$. EGFR is overexpressed in more than $60 \%$ of triple-negative breast cancers (TNBCs), as well as in non-small cell lung cancers (NSCLC), colorectal cancer (CRC), and glioblastoma $(3,4)$. It can activate several downstream signaling pathways related to cancer when the EGFR extracellular domain binds to its ligands. Given the important role of EGFR in multiple cancer processes such as proliferation and metastasis, various tyrosine kinase inhibitors (TKIs) and monoclonal antibodies (mAbs) have been developed for targeting EGFR in human cancers. Although EGFR-targeted therapies have shown success in certain cases, many patients fail to respond because tumors inevitably develop acquired resistance (5-7). Therefore, it is crucial to identify other treatments in the prevention of cancer.

One of the therapeutic methods that can meet this need is immunotoxins (ITs). ITs are antibody-cytotoxin chimeric molecules with specific cell killing ability (8). To date, unprecedented progress in treating hematological tumors has been achieved. Lumoxiti (moxetumomab pasudotox-tdfk) is a first-class anti-CD22 recombinant IT that fuses the binding domain of the anti-CD22 antibody to PE38 (9). It has been approved by the FDA as an intravenous fluid for the treatment of adult patients with recurrent or refractory hairy cell leukemia (HCL) (9). However, ITs are not as optimistic as hematologic malignancies in the treatment of solid tumors $(10,11)$. The capacity of IT to penetrate solid tumors (12), the generation of antitoxin antibodies $(9,13)$, and their clearance through kidney filtration (14) all limit the role of IT therapy in the treatment of solid tumors.

Nanoantibody, also known as single-domain antibody (sdAb), is the smallest known antigen-binding fragment available, preserving the full binding capacity of an intact antibody (15). Compared with conventional antibodies, the benefits of nanobodies include ease of expression, low molecular weights, good tissue penetration, high stability and solubility, and refolding ability; these benefits have granted their applications for the medical and biotechnological fields (15).

A novel typical type I ribosome-inactivating protein (RIP) known as cucurmosin (CUS) was isolated from the sarcocarp of Cucurbita moschata by our group with a determined DNA sequence and spatial structure (16). CUS can inhibit the growth 
of different human tumor cells in vitro and in vivo, including, but not limited to, PANC-1 (17), HepG2 (18), CFPAC-1 (19), and SW-1990 (20), but it presents low toxicity to normal cells. Compared with other RIPs, such as luffaculin and trichosanthin, the cytotoxicity of CUS is 4- to 7-fold stronger. These data indicated that CUS can be used as a toxic component of ITs targeted at tumor cells.

We previously reported an anti-EGFR nanobody 7D12-based recombinant immunotoxin rE/CUS (21), which can selectively kill EGFR ${ }^{+}$cells in vitro. $9 \mathrm{G} 8$ is another anti-EGFR nanobody with different epitope specificities compared with 7 D12 (22). Previous findings showed that 7D12 and 9G8 did not compete for binding to EGFR (22). To improve the binding ability of rE/CUS and creation of more potential ITs, the bispecific nanobody 7D12-9G8 was used instead of 7D12, which is capable of binding EGFR, and conjugated CUS using a flexible linker $(\mathrm{G} 4 \mathrm{~S})_{3}$ by genetic engineering methods. The bispecific recombinant IT known as Bs/CUS is more sensitive to cancer cell lines with EGFR expression and has a stronger cytotoxic effect than rE/CUS. The selectivity and cytotoxicity of Bs/CUS illustrate its potential as a novel candidate for treating EGFR-overexpressing tumors (22).

The aim of the current study was to examine a novel targeting EGFR recombinant immunotoxin CUS generated by fusing CUS to the EGFR-specific nanobody 7D12-9G8. The results indicated that Bs/CUS is a promising candidate that should be further evaluated as a cancer therapeutic for the treatment of EGFR-positive tumors.

\section{Materials and methods}

Reagents. Cetuximab was obtained from Merck. Goat $\mathrm{pAb}$ to human IgG (FITC) (ab81051; Abcam), Rb pAb to 6X His-tag ${ }^{\circledR}$ (FITC) (ab1206; Abcam), mouse-IgGK BP-HRP (sc-516102; Santa Cruz Biotechnology), and mouse anti-6X His Tag ${ }^{\circledR}$ antibody (ab18184; Abcam) were used in the present study. Goat-anti-mouse IgG-APC (550826; BD Bioscience), and FITC conjugate goat anti-mouse IgG (AMS.ASS1105-1000; Boster Biological Technology) were also used. Mouse anti-CUS antibody, 1G9, was produced by our laboratory. Escherichia coli strain BL21 (DE3), plasmid pET32a (Sangon Biotech), and Ni-NTA Sepharose FF (GE Healthcare) were utilized.

Cell lines. Human hepatoma cell line HepG2, human NSCLC cell line A549, and human CRC cell lines SW1116 and SW620 were obtained from the cell bank/stem cell bank of the Chinese Academy of Sciences. All cells were cultured in RPMI-1640 medium (Gibco; Thermo Fisher Scientific, Inc.) supplemented with $10 \%$ fetal bovine serum (Gibco; Thermo Fisher Scientific, Inc.). All the cell lines used were authenticated by short tandem repeat-based DNA profiling.

Production and characterization of recombinant IT. Anti-EGFR bispecific nanobody 7D12-9G8 was cloned into pET32a expression plasmid-containing CUS (designed Bs/CUS). The expression and purification of Bs/CUS were performed following previously described protocol (21).

Western blot analysis and 12\% SDS-PAGE gel were used to analyze the purified protein. The mouse-anti-His tag antibody and mouse anti-CUS antibody were used as primary antibodies, and mouse- $\mathrm{IgG}_{\mathrm{K}} \mathrm{BP}-\mathrm{HRP}$ was used as the secondary antibody.

EGFR expression and Bs/CUS binding capability. Cell surface EGFR expression of A549, HepG2, SW1116, and SW620 cells and binding ability of Bs/CUS were assessed by flow cytometry. To evaluate cell surface EGFR expression, cells were incubated with cetuximab and detected by anti-human-IgG (FITC). To verify Bs/CUS binding ability, $1 \mu \mathrm{g}$ of $\mathrm{rE} / \mathrm{CUS}$ and Bs/CUS was incubated with the abovementioned cell lines at $4^{\circ} \mathrm{C}$ for $30 \mathrm{~min}$, respectively. The mouse-anti-6X His tag antibody was added, followed by the detection antibody anti-mouse-IgG (APC), and analyzed by flow cytometry.

In vitro cytotoxicity assay. Sulforhodamine B (SRB) was applied to assess the cytotoxic activity of drugs as previously described (21). In brief, cells $\left(3 \times 10^{4} / \mathrm{ml}\right.$ per well) were added into 96-well plates in RPMI-1640 medium and incubated overnight at $37^{\circ} \mathrm{C}$ in $5 \% \mathrm{CO}_{2}$. CUS, 7D12-9G8, rE/CUS, Bs/CUS, and CUS+7D12-9G8 at different concentrations were added for 72 and $120 \mathrm{~h}$, respectively. Absorbance was measured by using an Epoch Microplate Reader (BioTek instrument, Inc.) at $515 \mathrm{~nm}$. Cytotoxic activity was defined by $\mathrm{IC}_{50}$ values.

Apoptosis assay. The FITC-Annexin V apoptosis kit (KeyGen Biotechnology Co., Ltd.) was used to evaluate the apoptotic effects of Bs/CUS on HepG 2 cells. Cells at a density of $4 \times 10^{4} / \mathrm{ml}$ per well were seeded in 6 -well plates, incubated overnight at $37^{\circ} \mathrm{C}$ and treated for $72 \mathrm{~h}$ with Bs/CUS or kept untreated (UT) at concentrations of $1,4,16$, and $64 \mathrm{nmol} / 1$. Subsequently, the cells were collected, centrifuged at $500 \mathrm{x} \mathrm{g}$ for $5 \mathrm{~min}$ at room temperature, counted, $5 \mu \mathrm{l}$ Annexin V-FITC and $5 \mu \mathrm{l}$ PI were added for $15 \mathrm{~min}$ at room temperature in the dark for staining, resuspended in $500 \mu \mathrm{l}$ binding buffer, and analyzed by flow cytometry (BD Biosciences).

Cell cycle analysis. Cell cycle changes induced by Bs/CUS treatment were studied by flow cytometry. In preparation for the cell cycle assay, HepG2 cells $\left(1 \times 10^{6}\right.$ cells/well) were seeded in 6-well plates and incubated overnight at $37^{\circ} \mathrm{C}$. The cells were then treated with Bs/CUS or kept untreated (UT) at concentrations of 1 and $4 \mathrm{nmol} / \mathrm{l}$ and incubated with serum-free medium for 24 and $48 \mathrm{~h}$, respectively. Cells were collected by EDTA-free trypsinization, fixed in $70 \%$ ethanol, and treated with PI solution $(50 \mu \mathrm{g} / \mathrm{ml} \mathrm{PI}$ and $100 \mu \mathrm{g} / \mathrm{ml}$ RNase A) (Sigma-Aldrich; Merck KGaA) for $30 \mathrm{~min}$ at $37^{\circ} \mathrm{C}$. Flow cytometry was used to analyze the cell cycle status of treated HepG2 cells.

Confocal microscopy. HepG2 cells were seeded in glass bottom cell culture dishes at a density of $1 \times 10^{5}$ cells per well and allowed to attach overnight. The next day, the cells were incubated with $5 \mu \mathrm{g} / \mathrm{ml}$ Bs/CUS for 3, 6, and $9 \mathrm{~h}$, respectively. The cells were washed with PBS and fixed with $4 \%$ paraformaldehyde for $15 \mathrm{~min}$ at room temperature and $0.5 \%$ Triton X-100 permeate for $15 \mathrm{~min}$. The cells were then washed with cold PBS and incubated in blocking buffer (PBS containing 5\% bovine serum albumin) at room temperature for $30 \mathrm{~min}$, followed by incubation with the primary antibody $1 \mathrm{G} 9$ at $4^{\circ} \mathrm{C}$ overnight. Cells were washed with cold PBS three times and incubated 

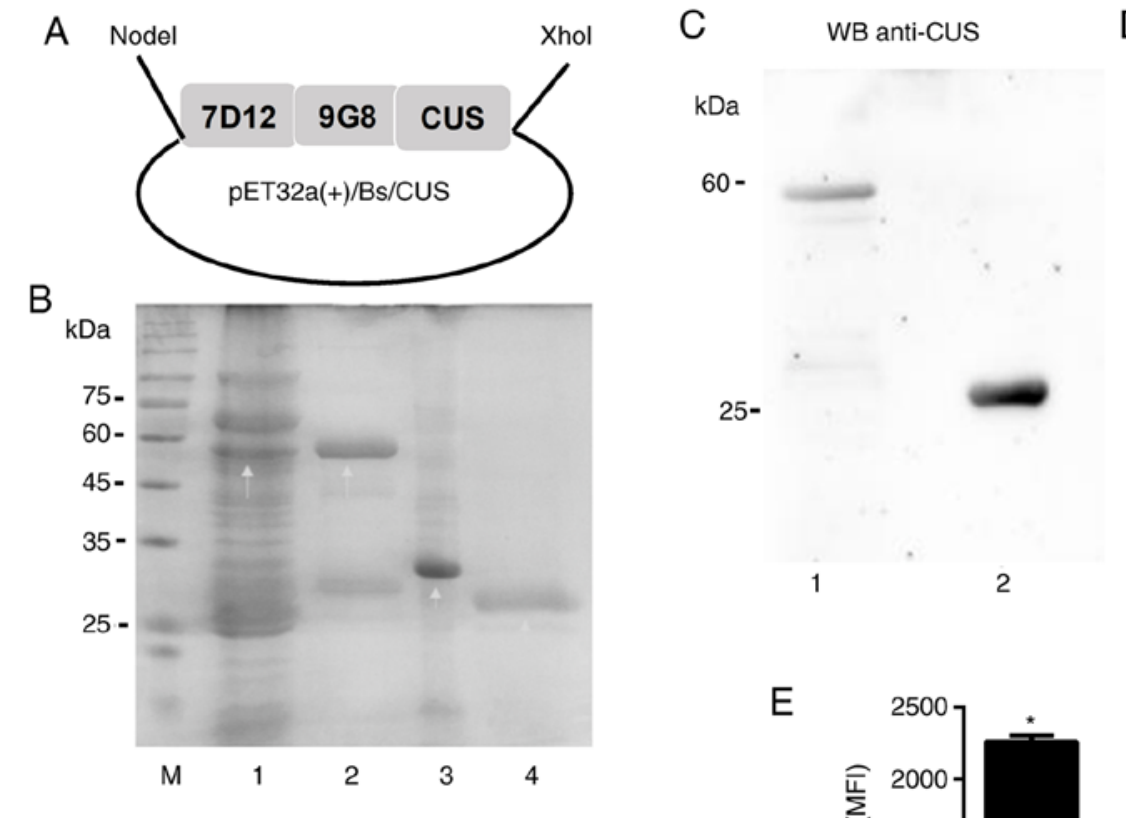

D WB anti-His
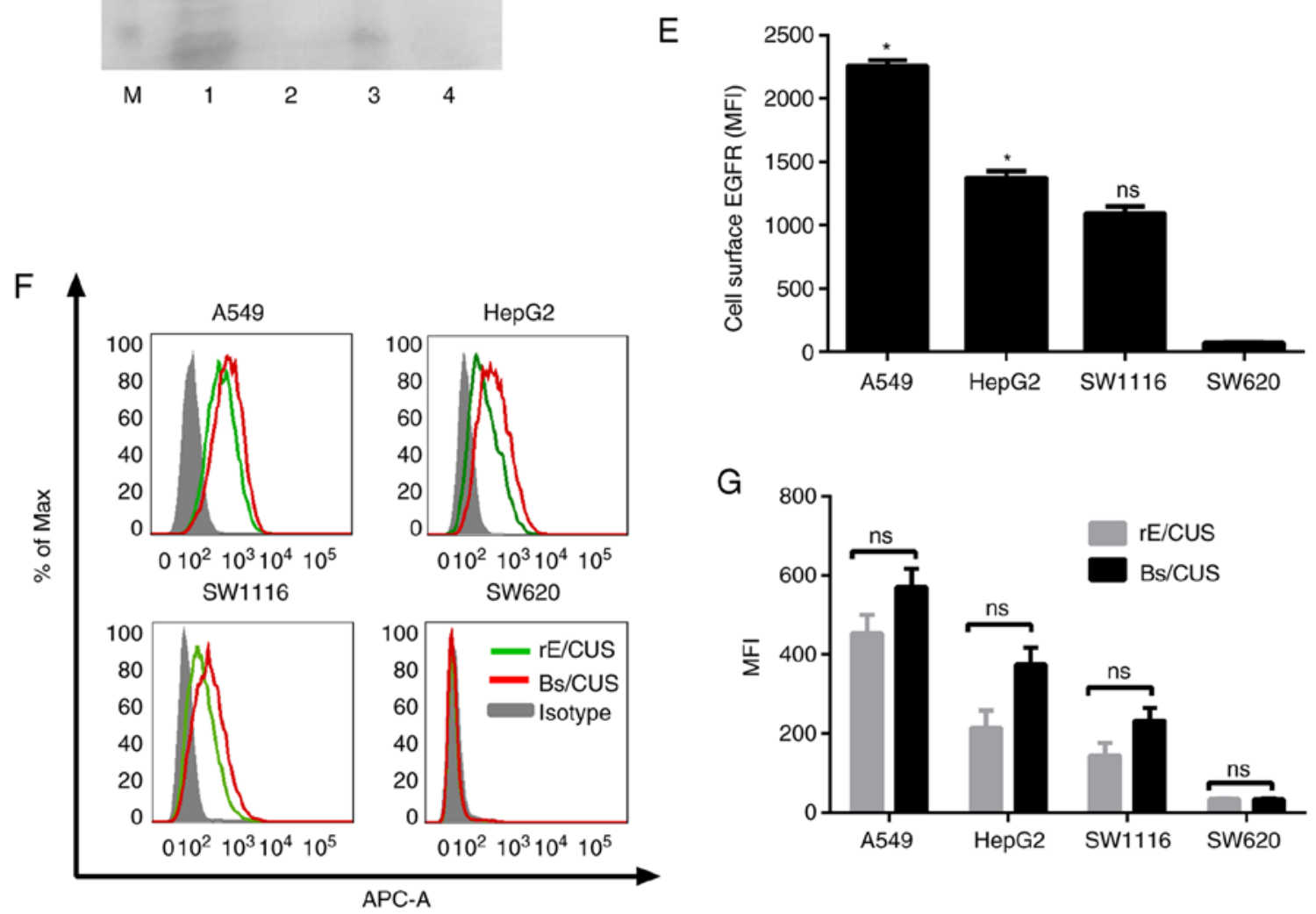

Figure 1. Expression and characterization of RIT. The pET32a(+)/Bs/CUS plasmid was transformed into E. coli BL21 (DE3) cells. (A) Schematic structure of pET32a(+)/Bs/CUS. (B) Use of 12\% SDS-PAGE under reduced conditions. M, protein marker; 1, E. coli BL21 (DE3) transformed with pET32a(+)/Bs/CUS induced by $1 \mathrm{mM}$ IPTG; 2, Bs/CUS purified by Ni-NTA column. 3, 7D12-9G8; 4, CUS. (C and D) Western blot confirmed the expression of Bs/CUS and CUS by using the primary antibodies against CUS and His tag, respectively. 1, Bs/CUS; 2, CUS. (E) EGFR expression on different cell lines. (F and G) Affinity of $\mathrm{Bs} / \mathrm{CUS}$ compared with that of rE/CUS by flow cytometry. MFI, mean fluorescence intensity. "P<0.05 vs. SW620.

with the indicated goat anti-mouse IgG (FITC) secondary antibody for $1 \mathrm{~h}$ at room temperature. DAPI was applied to stain the cell nuclei at room temperature for 15 min. Images were obtained by confocal microscopy using an LSM 710 system (Carl Zeiss) with 63x water C-Apochromat objective.

Statistical analysis. Each experiment was repeated in triplicate to determine the reproducibility of the results. Experimental data were presented as mean $\pm \mathrm{SD}$. A one-way ANOVA followed by Dunnett's multiple comparison test and Student's t-test were used to analyze the statistical comparisons. $\mathrm{P}<0.05$ was considered statistically significant.

\section{Results}

Generation and characterization of $B S / C U S$. The recombinant plasmid of pET32a(+)/Bs/CUS was constructed as shown in Fig. 1A. Bs/CUS and CUS were expressed in E. coli BL21 (DE3) and analyzed using 12\% SDS-PAGE gel (Fig. 1B). Western blot analysis was applied to confirm the recombinant proteins Bs/CUS and CUS by using 1G9 (Fig. 1C) and mouse-anti-His tag antibody (Fig. 1D), respectively. The protein bands were detected at 58 and $28 \mathrm{kDa}$, which were consistent with the expected weights of Bs/CUS and CUS, respectively. These results demonstrated that the bispecific 
Table I. $\mathrm{IC}_{50}$ values of cells treated with drugs for 72 and $120 \mathrm{~h}$.

\begin{tabular}{|c|c|c|c|c|c|c|}
\hline \multirow[b]{2}{*}{ Cell lines } & \multicolumn{6}{|c|}{$\mathrm{IC}_{50}($ Mean $\pm \mathrm{SD})$} \\
\hline & Bs/CUS (nmol/1) & rE/CUS (nmol/l) & CUS (nmol/l) & CUS+7D12-9G8 (nmol/l) & IT-1 & IT-2 \\
\hline A549 (72 h) & $0.018 \pm 0.011^{\mathrm{a}}$ & $1.300 \pm 0.712^{b}$ & $215 \pm 0.106$ & $257 \pm 0.097$ & 9760 & 102.3 \\
\hline A549 (120 h) & $0.008 \pm 0.001^{\mathrm{a}}$ & $0.623 \pm 0.172^{b}$ & $180 \pm 0.026$ & $170 \pm 0.031$ & 22500 & 288.9 \\
\hline HepG2 (72 h) & $0.025 \pm 0.011^{\mathrm{a}}$ & $2.386 \pm 0.624^{\mathrm{b}}$ & $244 \pm 0.033$ & $337 \pm 0.015$ & 9760 & 102.3 \\
\hline HepG2 (120 h) & $0.008 \pm 0.002^{\mathrm{a}}$ & $0.551 \pm 0.163^{b}$ & $109 \pm 0.009$ & $187 \pm 0.010$ & 13625 & 197.8 \\
\hline SW1116 (72 h) & $0.138 \pm 0.107^{\mathrm{a}}$ & $7.665 \pm 2.602^{b}$ & $192 \pm 0.023$ & $261 \pm 0.047$ & 1391 & 25.05 \\
\hline SW1116 (120 h) & $0.025 \pm 0.011^{\mathrm{a}}$ & $1.820 \pm 0.884^{\mathrm{b}}$ & $74 \pm 0.016$ & $72 \pm 0.007$ & 2960 & 40.66 \\
\hline SW620 (72 h) & $186.8 \pm 41.96^{\mathrm{a}}$ & $494.2 \pm 73.79^{\mathrm{b}}$ & $550 \pm 0.201$ & $471 \pm 0.089$ & 2.944 & 0.001 \\
\hline SW620 (120 h) & $88.89 \pm 5.71^{\mathrm{a}}$ & $305 \pm 19.83^{b}$ & $202 \pm 0.011$ & $169 \pm 0.023$ & 2.272 & 0.662 \\
\hline
\end{tabular}

${ }^{\mathrm{a}} \mathrm{P}<0.01$ vs. CUS; ${ }^{\mathrm{b}} \mathrm{P}<0.01$ vs. Bs/CUS. TI-1: Target index-1= $\mathrm{IC}_{50}{ }^{\mathrm{CUS}} / \mathrm{IC}_{50}{ }^{\mathrm{Bs} / \mathrm{CUS}} ;$ TI-2: Target index $-2=\mathrm{IC}_{50}{ }^{\mathrm{Cus}} / \mathrm{IC}_{50}{ }^{\mathrm{rE} / \mathrm{CUS}}$.

recombinant IT-Bs/CUS was successfully expressed in the E. coli prokaryotic expression system and purified.

The EGFR expression at the cell surface level was analyzed by flow cytometry using cetuximab as a primary antibody. As shown in Fig. 1E, A549 and HepG2 had the higher EGFR expression level $(\mathrm{P}<0.05)$, while SW1116 had relatively lower expression levels. SW620 cells were considered EGFR-negative cells.

Flow cytometry was applied to analyze the binding characteristic of Bs/CUS and compared with that of rE/CUS. Fig. $1 \mathrm{~F}$ and $\mathrm{G}$ show that the fluorescence intensity of Bs/CUS was higher than that of rE/CUS, indicating that Bs/CUS had stronger binding activity than $\mathrm{rE} / \mathrm{CUS}$. The rank order of the sensitivities of the cell lines to Bs/CUS in the EGFR expression level was A549>HepG2>SW1116>SW620, which was consistent with cetuximab. These results demonstrated that Bs/CUS retained the binding capacity and specificity with EGFR and was superior to rE/CUS.

Cytotoxicity in vitro. An SRB-based assay was applied to determine the specific cytotoxicity of Bs/CUS in vitro. The various tumor cell lines were incubated with decreasing concentrations of CUS, 7D12-9G8, rE/CUS, Bs/CUS, and CUS + 7D12-9G8 for 72 and $120 \mathrm{~h}$. The proteins were found to be cytotoxic in a dose-dependent manner on target cells (Fig. 2A). rE/CUS and Bs/CUS were significantly more cytotoxic than CUS alone $(\mathrm{P}<0.05)$. For the 72 -h experiments, the $\mathrm{IC}_{50}$ of $\mathrm{Bs} / \mathrm{CUS}$ to $\mathrm{SW} 1116$ was $>0.1 \mathrm{nmol} / 1$ but decreased to $0.02 \mathrm{nmol} / 1$ for A549 and HepG2 (Table I). At the 120 -h tests, the $\mathrm{IC}_{50}$ values of Bs/CUS to the abovementioned cell lines were 0.025, 0.008, and $0.008 \mathrm{nmol} / 1$, respectively, which decreased to 2 - to 5-fold lower than that at $72 \mathrm{~h}$ (Fig. 3A and Table I), suggesting a time-dependent cytotoxicity of Bs/CUS. Notably, the ability of Bs/CUS to inhibit protein synthesis increased by 70-100 times compared with the rE/CUS previously developed by our group. The highest target index of Bs/CUS was approximately 22,500, which indicated a 78-fold increase compared with that of $\mathrm{rE} / \mathrm{CUS}$.

After CUS and 7D12-9G8 were mixed at a molar ratio of 1:1, they were incubated with different tumor cells for 72 and 120 h. Results showed that the $\mathrm{IC}_{50}$ of CUS + 7D12-9G8 was consistent with CUS alone. This result suggested that Bs/CUS-inhibited protein synthesis was not a result of the simple synergy of the two drugs.

To determine whether the cytotoxic activity of Bs/CUS is specific, RIT was investigated with the non-target (EGFR') cell line SW620. As shown in Fig. 2 and Table I, the cytotoxicity of Bs/CUS did not increase in comparison with both CUS and CUS + 7D12-9G8.

Annexin V/PI assay was applied to determine whether the cytotoxicity of Bs/CUS is caused by apoptosis, and the data are shown in Fig. 2B and C. The results indicated a significant increase in early and late apoptosis in Bs/CUS in comparison with the untreated control. The apoptosis rate increased with the increasing concentration of Bs/CUS. The percentage of apoptosis was $22.45 \pm 0.21,69.60 \pm 0.14,66.50 \pm 0.42$ and $74.15 \pm 2.33$, respectively, in Bs/CUS-treated HepG2 at different concentrations. When the concentration of Bs/CUS was half that of $\mathrm{rE} / \mathrm{CUS}(8 \mathrm{nmol} / \mathrm{l})$, the apoptosis rate was higher than that of rE/CUS. Thus, Bs/CUS was found to be highly efficient in inducing apoptosis-mediated cell death.

Cell cycle analysis. HepG2 cells were treated with different doses of Bs/CUS (PBS, 1 and $4 \mathrm{nmol} / \mathrm{l}$ ) for 24 and $48 \mathrm{~h}$, and flow cytometric analysis was carried out to detect the cell cycle distribution via PI staining. For the $24 \mathrm{~h}$ test, it was found that Bs/CUS caused arrest in the G0/G1 phase and the $48 \mathrm{~h}$ test compared with the control group (Fig. 3). The proportion of cells incubated with $4 \mathrm{nmol} / \mathrm{l} \mathrm{Bs} / \mathrm{CUS}$ significantly increased in the G0/G1 phase and decreased in the proportion of cells in the $\mathrm{S}$ and $\mathrm{G} 2 / \mathrm{M}$ phases (10.58 and 4.81\%, respectively; $\mathrm{P}<0.0001)$.

Internalization of $B S / C U S$. Indirect immunofluorescence was applied to confirm the internalization of Bs/CUS in HepG2 cells. As shown in Fig. 4, the fluorescent signal was detected mainly on the cell surface treated with Bs/CUS for $3 \mathrm{~h}$. For $6 \mathrm{~h}$, signals started to accumulate in the cytoplasm, and the fluorescence signal was detected both in the cytoplasm and membrane. At incubation periods longer than $9 \mathrm{~h}$, the internalized Bs/CUS protein appeared as evenly distributed dots in the cytoplasm, while the cell membrane showed no luminescence, indicating that it had completely entered the cytoplasm. 

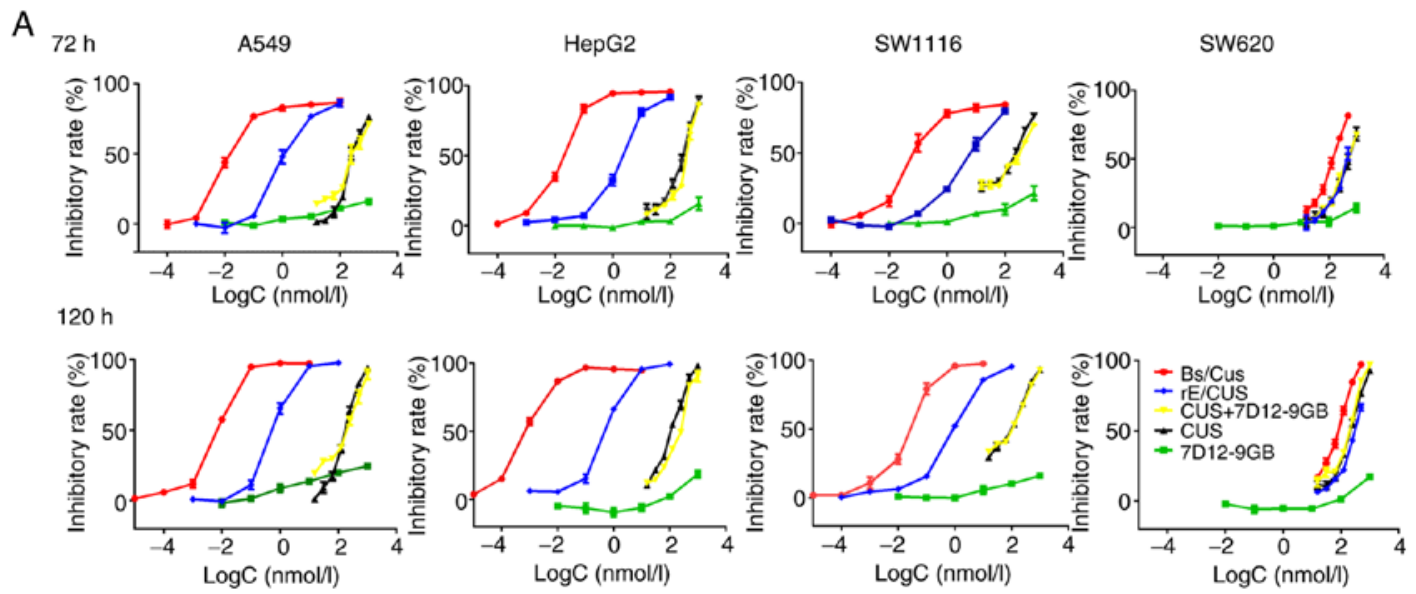

B
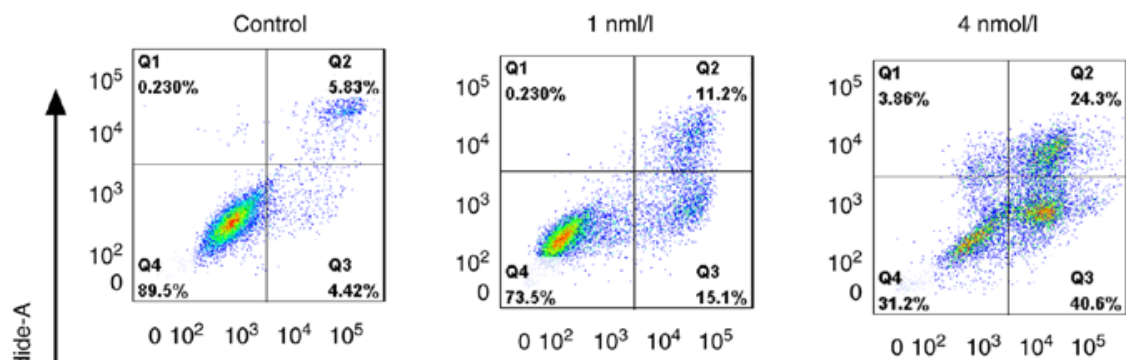

흘
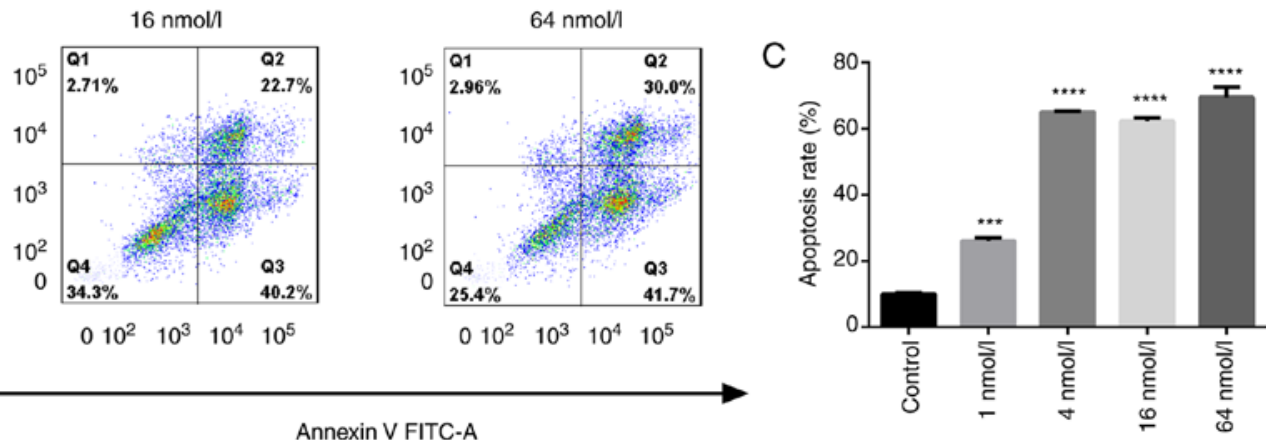

Figure 2. (A) Efficacy of drugs on different cancer cells in vitro by SRB assay. Cells exposed to Bs/CUS, rE/CUS, CUS, 7D12-9G8, and CUS + 7D12-9G8 for 72 and $120 \mathrm{~h}$. (B) The apoptotic activity of Bs/CUS in HepG2 cells, and results of quantitative analysis $(\mathrm{C}){ }^{* * *} \mathrm{P}<0.001,{ }^{* * * *} \mathrm{P}<0.0001$ vs. control.

\section{Discussion}

EGFR is overexpressed in various tumors and is associated with cancer initiation, progression, and poor prognosis, by mutations, gene amplification, or both through constitutive EGFR activation $(3,4)$. Cancer therapies targeting EGFR, such as TKIs and mAbs, have been developed as standard therapies for several cancers including but not limited to NSCLC and CRC (23-25). The main limitation of treatments targeting EGFR is the appearance of acquired resistance. Immunotoxins function not by suppressing receptor-mediated signaling but by directly killing the cells. In this study, CUS, the toxin moiety used is a typical type I RIP. It can hydrolyze the N-glycosidic bond at A4324 on the 28S rRNA of eukaryotic cells, irreversibly inactivating the ribosomal 60S subunit, which halts protein synthesis. Thus, there can be less chance for tumor cells to upregulate rescue mutations or alternative signaling pathways to resist immunotoxin therapy (26).

Given the importance of EGFR in solid tumors, we constructed a recombinant IT by using the anti-EGFR bispecific nanobody 7D12-9G8 fused to a toxin known as Bs/CUS and demonstrated the anti-tumor activity of the IT Bs/CUS in vitro. The conventional recombinant IT molecules are constructed by fusion of the toxin with $\mathrm{scFv}$ or $\mathrm{dsFv}$, which often have the problems of stability, water solubility, and aggregation (27). Nevertheless, the IT Bs/CUS based on bispecific nanobody did not have the abovementioned problems, possibly due to the beneficial outcomes of the nanobody, so it could be directly expressed in the E. coli system as soluble proteins.

According to previous findings, when the various tumor cell lines were incubated with decreasing concentrations of CUS for 24, 48, 72, 96, and $120 \mathrm{~h}$, the proteins were found to be cytotoxic in a time-dependent manner on target cells (17-19). Results of the cytotoxicity assay in vitro showed that the killing ability of Bs/CUS on different tumor cells was time-dependent under the conditions of 72 and $120 \mathrm{~h}$ incubation. This result and incubation time are consistent with the research results of other immunotoxins using CUS as a toxic agent in our laboratory $(28,29)$. 

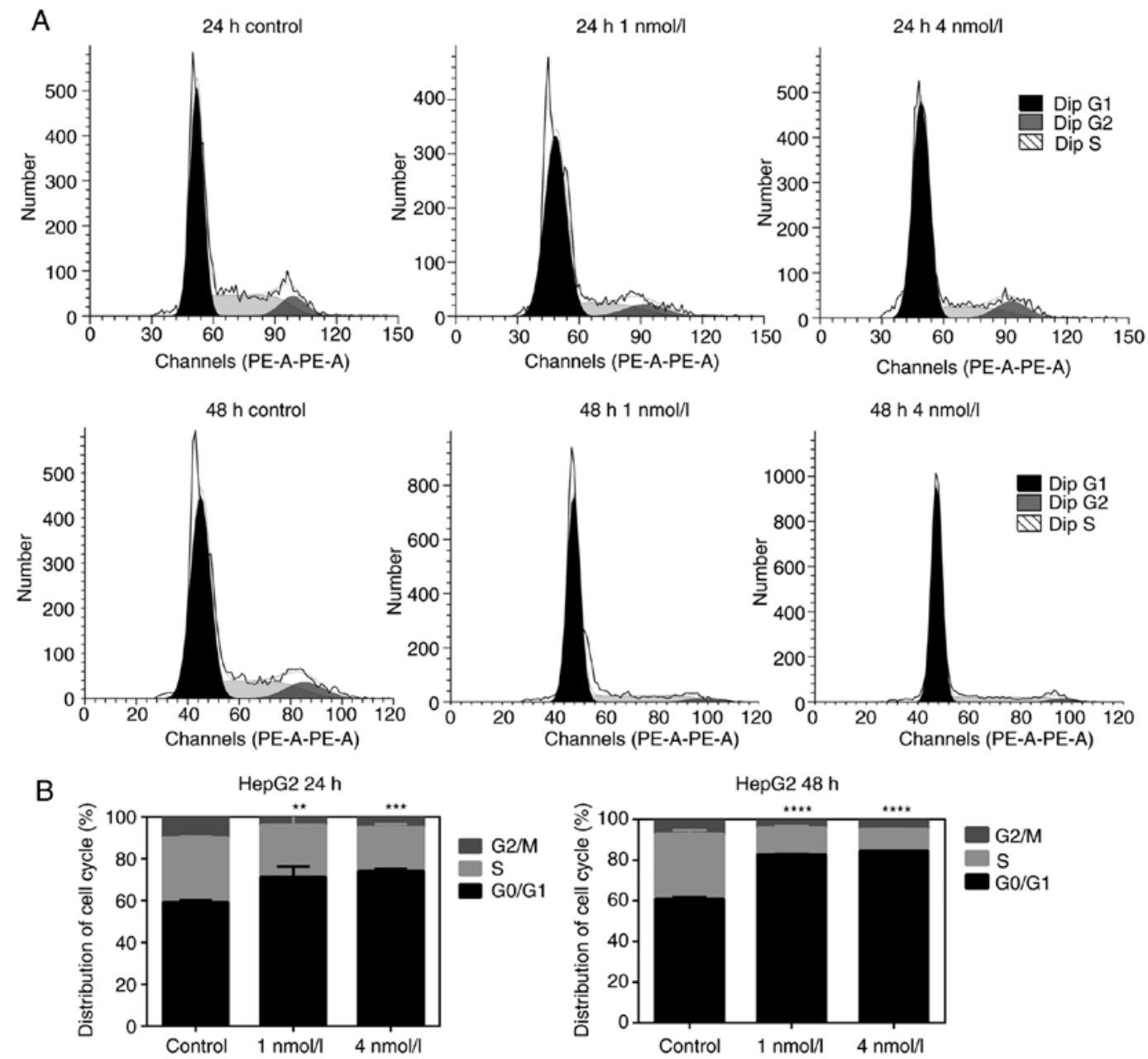

Figure 3. Bs/CUS caused cell cycle arrest in HepG2 cancer cells. (A) Flow cytometric analysis detected cell cycle distribution. (B) Quantitative analysis. ${ }^{* *} \mathrm{P}<0.01,{ }^{* * *} \mathrm{P}<0.001,{ }^{* * * *} \mathrm{P}<0.0001$ vs. control.

On the basis of the combination tests of flow cytometry, SRB cytotoxicity, and apoptosis assay, our results showed that the IT Bs/CUS had higher EGFR binding capacity and binding specificity than $\mathrm{rE} / \mathrm{CUS}$. These results were consistent with previous reports showing that the bi-paratopic 9G8-7D12 molecule had a higher affinity than 7D12 and 9G8 alone, respectively, due to their different binding sites on the EGFR receptor (22). However, there was no statistical difference between the affinity of $\mathrm{rE} / \mathrm{CUS}$ and Bs/CUS. The binding ability of Bs/CUS was lower than the chemically-linked conjugates, T-CUS ${ }_{245 \mathrm{C}}$ and D-CUS ${ }_{245 \mathrm{C}}$, which were constructed by our group. Therefore, future studies are necessary to understand the Kd value of Bs/CUS and further improve it. A comparison with other bispecific ITs also reflected the high potency against tumors of Bs/CUS, such as dDT2219, an RIT with an $\mathrm{IC}_{50}$ range of 0.23-1.03 nmol/1 against B-cell malignancies (30).

Nevertheless, we have verified that Bs/CUS exerts its function of killing tumor cells through the apoptotic pathway by annexin V/phycoerythrin-based apoptosis assay. To make the results solid, more verification experiments such as CCK8, DraQ7, and immunofluorescent staining using apoptosis-related antibody should be utilized in subsequent investigations. In addition, in the present study, we preliminarily measured that Bs/CUS induced HepG2 cell arrest in the
G0/G1 phase; however, the molecular mechanism of cell cycle arrest involved in Bs/CUS remains to be verified.

Given that the rE/CUS has a molecular weight of approximately $42 \mathrm{kDa}$, it could be cleared rapidly in vivo via the kidneys. Although Bs/CUS is an IT based on the fusion of bispecific nanobody with a molecular weight of $58 \mathrm{kDa}$, it may also face the above problem due to its size under the glomerular filtration threshold (70 kDa) (31). Accumulating evidence from multiple trials indicated that binding to albumin is an exceptional option to prolong the in vivo half-life of small proteins $(14,32,33)$. Thus, future studies are necessary to verify the effectiveness of Bs/CUS with the albumin-binding domain in vivo.

Taken together, Bs/CUS demonstrates high cytotoxicity and selectivity on EGFR-positive cancer cells, indicating that it could be a promising candidate and it should be further evaluated as a cancer therapeutic for the treatment of EGFR-positive tumors.

\section{Acknowledgements}

Not applicable.

\section{Funding}

This research was funded by the National Science Foundation of China (no. 30772587), the Natural Science Foundation of 


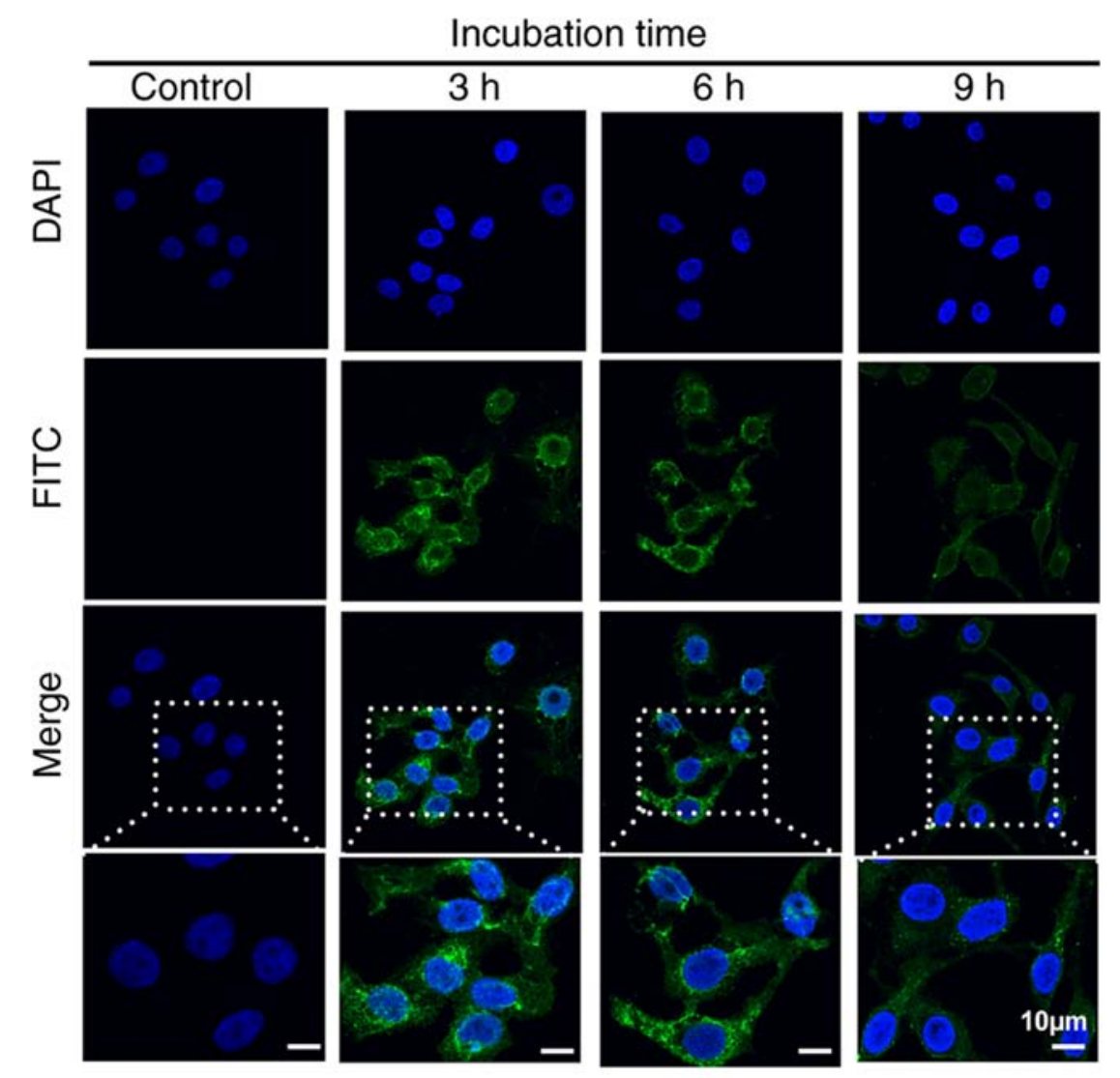

Figure 4. Internalization of Bs/CUS at 3, 6, and $9 \mathrm{~h}$ in HepG2 cells. Cells were incubated with FITC-goat anti-mouse IgG (green), which visualizes anti-CUS antibody. Nuclei were counterstained with DAPI (blue). Scale bar, $10 \mu \mathrm{m}$.

Fujian Province (no. 2016J01769), the Fujian Province Health and Family Planning Scientific Research Talent Training Project (no. 2018-CX-40), and Fujian Province Student Innovation and Entrepreneurship Project (no. 201810392045).

\section{Availability of data and materials}

The datasets used and analyzed during the current study are available from the corresponding author on reasonable request.

\section{Authors' contributions}

$\mathrm{CZ}$ and $\mathrm{YC}$ contributed to study concept and design, acquisition of data, analysis and interpretation of data, and drafting of the manuscript; XD performed the experiments; JW conducted additional experiments; YL contributed to the statistical analysis; HZ, ML, and JL contributed to the study concept, study supervision and critical revision of the manuscript; JX contributed to the study concept and design, study supervision and critical revision of the manuscript. All authors read and approved the final manuscript.

\section{Ethics approval and consent to participate}

Not applicable.

\section{Patient consent for publication}

Not applicable.

\section{Competing interests}

The authors declare that they have no competing interests.

\section{References}

1. Garrett TPJ, McKern NM, Lou M, Elleman TC, Adams TE, Lovrecz GO, Zhu HJ, Walker F, Frenkel MJ, Hoyne PA, et al: Crystal structure of a truncated epidermal growth factor receptor extracellular domain bound to transforming growth factor alpha. Cell 110: 763-773, 2002.

2. Ogiso H, Ishitani R, Nureki O, Fukai S, Yamanaka M, Kim JH, Saito K, Sakamoto A, Inoue M, Shirouzu M and Yokoyama S: Crystal structure of the complex of human epidermal growth factor and receptor extracellular domains. Cell 110: 775-787, 2002.

3. Ciardiello $\mathrm{F}$ and Tortora G: EGFR antagonists in cancer treatment. N Engl J Med 358: 1160-1174, 2008.

4. Furnari FB, Fenton T, Bachoo RM, Mukasa A, Stommel JM, Stegh A, Hahn WC, Ligon KL, Louis DN, Brennan C, et al: Malignant astrocytic glioma: Genetics, biology, and paths to treatment. Genes Dev 21: 2683-2710, 2007.

5. Morgillo F, Della Corte CM, Fasano M and Ciardiello F: Mechanisms of resistance to EGFR-targeted drugs: Lung cancer. ESMO Open 1: e000060, 2016.

6. Misale S, Yaeger R, Hobor S, Scala E, Janakiraman M, Liska D, Valtorta E, Schiavo R, Buscarino M, Siravegna G, et al: Emergence of KRAS mutations and acquired resistance to anti-EGFR therapy in colorectal cancer. Nature 486: 532-536, 2012.

7. Lynch TJ, Bell DW, Sordella R, Gurubhagavatula S, Okimoto RA, Brannigan BW, Harris PL, Haserlat SM, Supko JG, Haluska FG, et al: Activating mutations in the epidermal growth factor receptor underlying responsiveness of non-small-cell lung cancer to gefitinib. N Engl J Med 350: 2129-2139, 2004. 
8. Alewine C, Hassan R and Pastan I: Advances in anticancer immunotoxin therapy. Oncologist 20: 176-185, 2015.

9. Kreitman RJ, Tallman MS, Robak T, Coutre S, Wilson WH, Stetler-Stevenson M, Fitzgerald DJ, Lechleider R and Pastan I: Phase I trial of anti-CD22 recombinant immunotoxin moxetumomab pasudotox (CAT-8015 or HA22) in patients with hairy cell leukemia. J Clin Oncol 30: 1822-1828, 2012.

10. Hassan R, Bullock S, Premkumar A, Kreitman RJ, Kindler H Willingham MC and Pastan I: Phase I study of SS1P, a recombinant anti-mesothelin immunotoxin given as a bolus I.V. infusion to patients with mesothelin-expressing mesothelioma, ovarian, and pancreatic cancers. Clin Cancer Res 13: 5144-5149, 2007

11. Kreitman RJ, Hassan R, Fitzgerald DJ and Pastan I: Phase I trial of continuous infusion anti-mesothelin recombinant immunotoxin SS1P. Clin Cancer Res 15: 5274-5279, 2009.

12. Mason-Osann E, Hollevoet K, Niederfellner G and Pastan I Quantification of recombinant immunotoxin delivery to solic tumors allows for direct comparison of in vivo and in vitro results. Sci Rep 5: 10832, 2015

13. Mazor R, Crown D, Addissie S, Jang Y, Kaplan G and Pastan I: Elimination of murine and human T-cell epitopes in recombinant immunotoxin eliminates neutralizing and anti-drug antibodies in vivo. Cell Mol Immunol 14: 432-442, 2017.

14. Dennis MS, Zhang M, Meng YG, Kadkhodayan M, Kirchhofer D, Combs D and Damico LA: Albumin binding as a general strategy for improving the pharmacokinetics of proteins. J Biol Chem 277: 35035-35043, 2002

15. Siontorou CG: Nanobodies as novel agents for disease diagnosis and therapy. Int J Nanomedicine 8: 4215-4227, 2013.

16. Hou X, Meehan EJ, Xie J, Huang M, Chen M and Chen L: Atomic resolution structure of cucurmosin, a novel type 1 ribosome-inactivating protein from the sarcocarp of Cucurbita moschata. J Struct Biol 164: 81-87, 2008.

17. Zhang B, Huang H, Xie J, Xu C, Chen M, Wang C, Yang A and Yin Q: Cucurmosin induces apoptosis of BxPC-3 human pancreatic cancer cells via inactivation of the EGFR signaling pathway. Oncol Rep 27: 891-897, 2012.

18. Xie J, Que W,Liu H,Liu M, Yang A and Chen M: Anti-proliferative effects of cucurmosin on human hepatoma HepG2 cells. Mol Med Rep 5: 196-201, 2012.

19. Xie J, Wang C, Zhang B, Yang A, Yin Q, Huang H and Chen M: Cucurmosin induces the apoptosis of human pancreatic cancer CFPAC- 1 cells by inactivating the PDGFR- $\beta$ signalling pathway. Pharmacol Rep 65: 682-688, 2013.

20. Xie J, Wang C, Yang A, Zhang B, Yin Q, Huang H and Chen M: Cucurmosin kills human pancreatic cancer SW-1990 cells in vitro and in vivo. Anticancer Agents Med Chem 13: 952-956, 2013.

21. Deng C, Xiong J, Gu X, Chen X, Wu S, Wang Z, Wang D, Tu J and Xie J: Novel recombinant immunotoxin of EGFR specific nanobody fused with cucurmosin, construction and antitumor efficiency in vitro. Oncotarget 8: 38568-38580, 2017.
22. Roovers RC, Vosjan MJ, Laeremans T, el Khoulati R, de Bruin RC, Ferguson KM, Verkleij AJ, van Dongen GA and van Bergen en Henegouwen PM: A biparatopic anti-EGFR nanobody efficiently inhibits solid tumour growth. Int J Cancer 129: 2013-2024, 2011.

23. Greenhalgh J, Dwan K, Boland A, Bates V, Vecchio F, Dundar Y, Jain $P$ and Green JA: First-line treatment of advanced epidermal growth factor receptor (EGFR) mutation positive non-squamous non-small cell lung cancer. Cochrane Database Syst Rev: CD010383, 2016

24. Jean GW and Shah SR: Epidermal growth factor receptor monoclonal antibodies for the treatment of metastatic colorectal cancer. Pharmacotherapy 28: 742-754, 2008.

25. Lee JJ and Chu E: First-line use of anti-epidermal growth factor receptor monoclonal antibodies in metastatic colorectal cancer. Clin Colorectal Cancer 2 (Suppl 6): S42-S46, 2007.

26. Simon N and FitzGerald D: Immunotoxin therapies for the treatment of epidermal growth factor receptor-dependent cancers. Toxins (Basel) 8: 137, 2016

27. Cheung LS, Fu J, Kumar P, Kumar A, Urbanowski ME, Ihms EA, Parveen S, Bullen CK, Patrick GJ, Harrison R, et al: Second-generation IL-2 receptor-targeted diphtheria fusion toxin exhibits antitumor activity and synergy with anti-PD-1 in melanoma. Proc Nat Acad Sci U S A 116: 3100-3105, 2019.

28. Xiong J, Zhang C, Wu S, Gu X, Cai Y, Xu C, Chen Z, Sun J, Wu X, You X, et al: Recombinant cucurmosin-based immunotoxin targeting HER-2 with potent in vitro anti-cancer cytotoxicity. Biochem Biophys Res Commun 513: 15-21, 2019.

29. Zhang C, Xiong J, Lan Y, Wu J, Wang C, Huang Z, Lin J and Xie J: Novel cucurmosin-based immunotoxin targeting programmed cell death 1-ligand 1 with high potency against human tumor in vitro and in vivo. Cancer Sci 111: 3184-3194, 2020.

30. Schmohl JU, Todhunter D, Taras E, Bachanova V and Vallera DA: Development of a deimmunized bispecific immunotoxin dDT2219 against B-cell malignancies. Toxins (Basel) 10: 32, 2018.

31. Sanz L, Blanco B and Alvarez-Vallina L: Antibodies and gene therapy: Teaching old 'magic bullets' new tricks. Trends Immunol 25: 85-91, 2004.

32. Roovers RC, Laeremans T, Huang L, De Taeye S, Verkleij AJ, Revets H, de Haard HJ and van Bergen en Henegouwen PMP: Efficient inhibition of EGFR signaling and of tumour growth by antagonistic anti-EFGR nanobodies. Cancer Immunol Immunother 56: 303-317, 2007.

33. Tijink BM, Laeremans T, Budde M, Stigter-van Walsum M, Dreier T, de Haard HJ, Leemans CR and van Dongen GAMS: Improved tumor targeting of anti-epidermal growth factor receptor Nanobodies through albumin binding: Taking advantage of modular nanobody technology. Mol Cancer Ther 7: 2288-2297, 2008.

This work is licensed under a Creative Commons Attribution-NonCommercial-NoDerivatives 4.0 International (CC BY-NC-ND 4.0) License. 